\title{
Multiple cerebral fusiform aneurysms involving the posterior and anterior circulation including the anterior cerebral artery: a case report
}

\author{
Sumit Das, Tibor Valyi-Nagy \\ Department of Pathology, University of Illinois at Chicago, United States
}

\begin{abstract}
Multiple fusiform aneurysms occurring in a single patient are very uncommon. Fusiform aneurysms affecting the anterior circulation are extremely rare and reported cases involve the middle cerebral artery and internal carotid artery. We report here a case of a 55-year-old African American male who had a history of multiple strokes and who was found unresponsive prior to his latest hospital admission. Neuroimaging was remarkable for multiple fusiform aneurysms involving segments of the anterior, middle and posterior cerebral arteries as well as subarachnoid and intraparenchymal hemorrhage. Unfortunately even after aggressive medical management, the patient did not show any signs of recovery and further management entailed comfort care measures only. The patient passed away shortly thereafter. Post-mortem examination confirmed the presence of multiple fusiform aneurysms with involvement of both the posterior and anterior circulation including the anterior cerebral artery as well as subarachnoid and intraparenchymal hemorrhage. Interestingly, there was no apparent site of aneurysm rupture identified to explain the hemorrhage. Our case is unusual in that the multiple fusiform aneurysms were found to extensively involve both the anterior and posterior circulation. Furthermore, our case demonstrates involvement of the anterior cerebral artery by fusiform aneurysms, which is also a very rare event.
\end{abstract}

Key words: aneurysm, multiple, hemorrhage, risk factors, pathogenesis.

\section{Introduction}

An aneurysm is a focal dilation of the arterial wall. The majority of aneurysms are of a saccular type. Fusiform aneurysms are nonsaccular dilations involving the entire arterial wall for a short distance [12]. They have different pathological features, anatomic distribution and natural histories [3]. They are formed from ectatic and tortuous basal arteries and typically arise in the middle part of the basilar artery [12].
Potential causes of fusiform aneurysms include atherosclerosis, dissection, disorders of collagen, and infection. Fusiform aneurysms affecting the anterior circulation are extremely rare and reported cases involve the middle cerebral artery and internal carotid artery [12]. Multiple fusiform aneurysms occurring in a single patient are also very uncommon [6,7]. We present here an unusual case of a patient with multiple fusiform aneurysms involving both the posterior and anterior circulation including the anterior cerebral artery with 
pathology confirmed on post-mortem examination. Our case is aimed to raise awareness of the possibility of diffuse involvement of the circle of Willis blood vessels by fusiform aneurysms and also describes the rare involvement of the anterior cerebral artery by this type of aneurysm.

\section{Case report}

A 55-year-old African American male with a past medical history of hypertension and multiple episodes of stroke first presented at an outside hospital with acute onset of right-sided extremity weakness, right facial droop, and slurred speech. Neurological examination was notable for mild dysarthria, expressive aphasia, mild facial droop, and clonus in the right ankle. Neuroimaging performed at the outside hospital revealed an infarction in the vascular territory of the left anterior cerebral artery. CT angiogram revealed multiple fusiform aneurysms along with severe stenosis of the right $M 2$ and left $M 2$ segments, occlusion of the right $\mathrm{A} 2$ and $\mathrm{A} 1$ segments, and intracranial portion of the left vertebral artery. The patient underwent pipeline stenting of the right P2 segment.

Six months later, the patient was found unresponsive at his home with surrounding evidence of emesis. He was subsequently transported to the outside hospital where he was intubated. CT angiogram revealed subarachnoid hemorrhage within the basilar cisterns and intraparenchymal hemorrhage involving the pons, midbrain and right basal ganglia. At this point the patient was transferred to our hospital for neurosurgical evaluation and management. Treatment for his aneurysms was held off pending improvement of his neurologic status. Unfortunately no such recovery was noted. One week later, the patient's family opted to switch the mode of care to comfort measures. The next morning he was noted to be in asystole. Pupils were fixed and dilated with no heart or lung sounds. No spontaneous movements were appreciated either. He was subsequently pronounced deceased.

\section{Post-mortem examination}

General autopsy was notable for moderate calcific atherosclerotic deposits in the abdominal aorta and bilateral common iliac arteries, cardiomegaly with globular shaped heart and bilateral ventricular hypertrophy along with moderate occlusion of the left anterior descending coronary artery.

\section{Neuropathologic examination}

The whole brain was examined both during the general autopsy and after formalin fixation. Extensive subarachnoid hemorrhage was identified predominantly over the ventral aspect of the brain but also involving parts of the cerebral convexity and cerebellar hemisphere. Dissection of the circle of Willis vessels confirmed the presence of multiple unruptured fusiform aneurysms involving segments of the anterior,
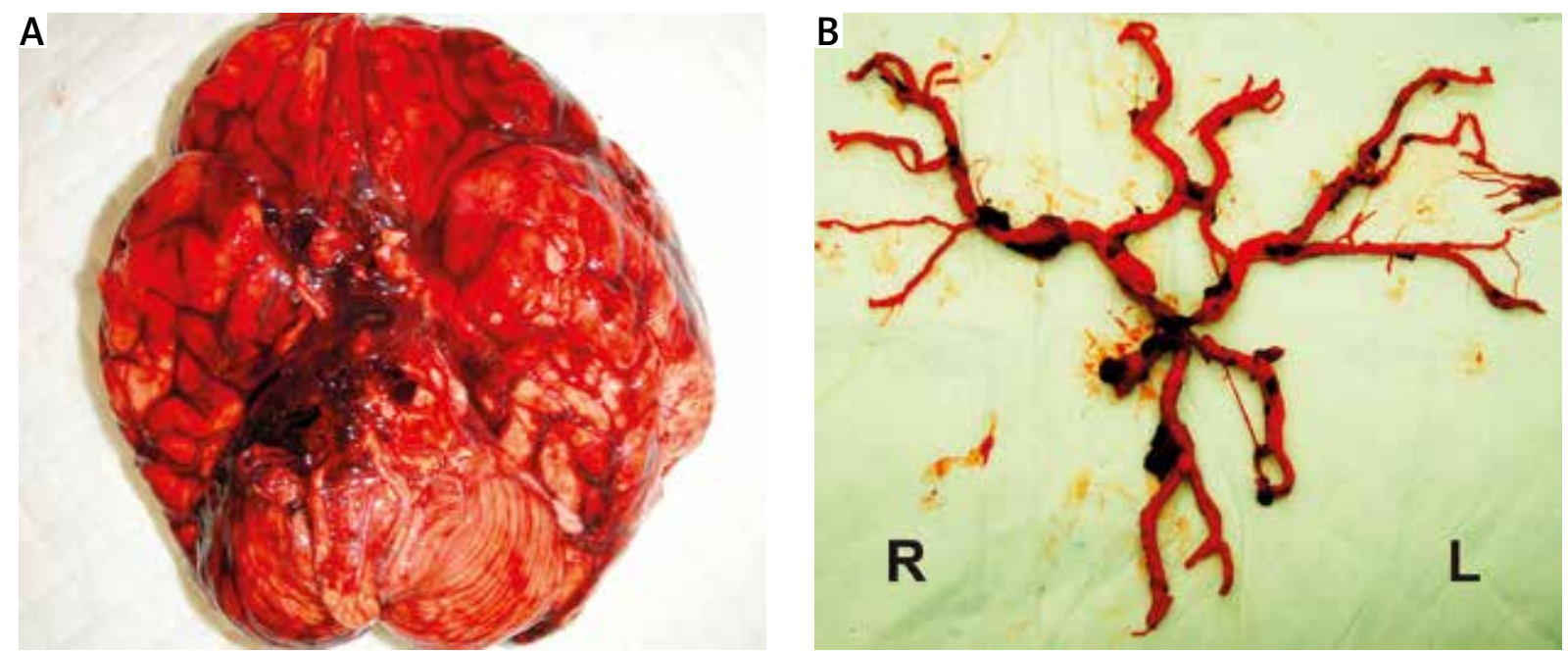

Fig. 1. A) Unfixed brain showing diffuse subarachnoid haemorrhage involving the ventral aspect of the brain including the brainstem and cerebellum. B) Vessels of the circle of Willis dissected out of the unfixed brain demonstrating multiple fusiform aneurysms, including involvement of the anterior cerebral artery. 
middle and posterior cerebral arteries as well as the basilar artery (Fig. 1). Severe atherosclerosis was also noted throughout the circle of Willis blood vessels.

Coronal sectioning of the cerebrum revealed bilateral intraparenchymal hemorrhage extending to the lateral ventricles. Hemorrhagic infarcts were noted in the anterior-medial aspect of the frontal lobe extending to the posterior parietal lobe and inferiorly to the basal ganglia. Portions of the thalamus and brainstem were also involved by hemorrhage. Intraparenchymal hemorrhage was also seen in the right cerebellum.

Microscopic sections confirmed the presence of aneurysms with the aid of elastic staining, which showed focal loss of the internal elastic lamina. The walls of the blood vessels involved by the aneurysms showed prominent atherosclerotic changes in the form of cholesterol clefts, scattered calcification and intimal hyperplasia. Prominent arteriolosclerosis was noted throughout sections of the cerebral hemisphere.

Subacute to chronic infarcts were observed in the sampled sections of the frontal cortex and mesiotemporal region. Sections of the midbrain and pons revealed extensive hemorrhagic infarcts.

\section{Discussion}

Fusiform aneurysm is a localized or diffuse dilation of an artery involving intima, media and adventitia.

A

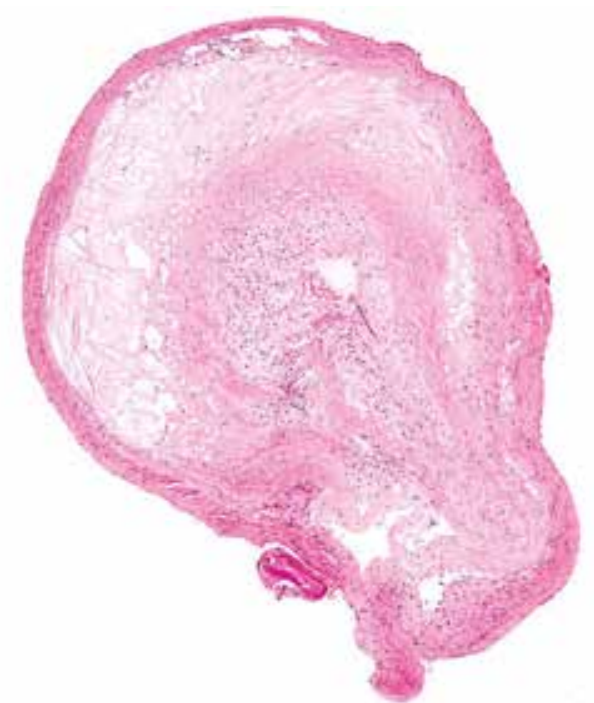

It is formed from ectatic, often tortuous, basal arteries. Most aneurysms occur in the basilar artery but are rare in the anterior circulation. When they do occur in the later, they are most commonly found in the middle cerebral artery and internal carotid artery [12]. Fusiform aneurysms that involve the anterior cerebral artery as in our patient are also extremely rare $[1,2,4]$. Typical presentations of fusiform aneurysms include brain stem and/or cranial nerve compression (basilar artery aneurysm), ischemia secondary to thrombosis, brain stem/cerebellar infarcts with a basilar artery aneurysm or hemorrhage.

Although definite risk factors for multiple aneurysms have not been clearly identified, reported associations include older age, female sex, hypertension, and cigarette smoking [8]. As previously mentioned, our patient had a long history of hypertension. Proposed etiologic factors of fusiform aneurysms include atherosclerosis, vessel dissection, collagen vascular disease, and fibromuscular dysplasia [13].

It has been suggested that the initial event in formation of a fusiform aneurysm is lipid deposition in and below the intima leading to disruption of the internal elastic membrane and infiltration of the muscular wall $[5,13]$. The resultant atrophy of the elastic substance and musculature then leads to tortuosity of the vessel due to intravascular pressure causing the ectatic vessel to expand in diameter and length. The increasing luminal diameter results in turbulent blood flow, which in turn can lead to thrombus for-

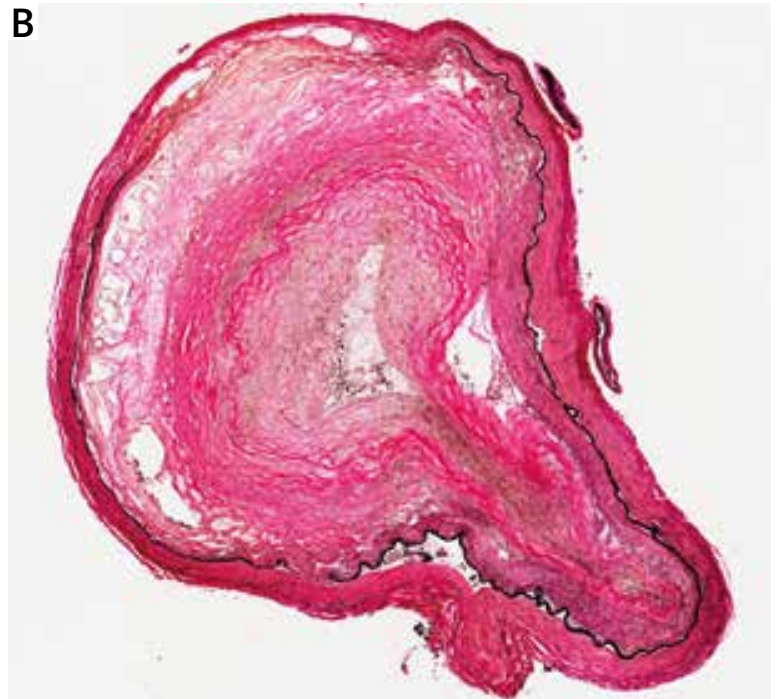

Fig. 2. A) Hematoxylin and eosin stained section from one of the major vessels demonstrating circumferential bulging and atheroma. B) Elastic stain demonstrating focal loss of internal elastic lamina. 
mation resulting in further intimal disruption and vessel distortion $[5,13,14]$. In our patient, multiple cerebral vessels were involved by significant atherosclerosis, which may have been the main pathogenic factor in the development of the observed fusiform aneurysms by causing significant weakening of the vessel walls. Histologic examination of the vessels involved by fusiform aneurysms also revealed significant atherosclerotic changes in the form of cholesterol clefts, calcification and intimal hyperplasia. We did not find any pathologic evidence of arterial dissection or any apparent collagen deposition within the walls of the blood vessels. The absence of obvious collagen deposition within the arterial wall made fibromuscular dysplasia or collagen vascular disease less likely factors in the formation of fusiform aneurysms in our patient.

Day (2003) conducted a review of 102 cases of spontaneous fusiform aneurysms based on which he proposed that spontaneous fusiform MCA aneurysms usually develop as a result of arterial dissection. He also suggested that spontaneous fusiform aneurysms in the anterior circulation, especially those involving the MCA, are more often found in younger patients. His review did not include aneurysms associated with trauma, infection, inflammation, and neoplasia. The study also did not touch on fusiform aneurysms occurring in the anterior cerebral artery territory. This raises the question of whether the clinical course of fusiform aneurysms differs depending on where in the anterior circulation they occur and if the mode of management differs in any way.

There are also very few reports of multiple fusiform aneurysms diffusely involving the cerebral vessels as they did in our patient [9]. The clinical course of such extensive involvement is therefore not fully understood and may warrant further study, which in turn can help guide future management and follow-up.

Hemorrhage resulting from rupture of fusiform aneurysms is rare although it has been suggested that fusiform aneurysms of the vertebral artery and internal carotid artery frequently present with hemorrhage [3]. In our case, a site of aneurysmal rupture was not found, leading us to presume that our patient's subarachnoid and intraparenchymal hemorrhages were likely secondary to uncontrolled hypertension.

In summary, we report here a very unusual case of multiple fusiform aneurysms affecting both the posterior and anterior circulation in a single patient with involvement of the anterior cerebral artery, which can pose a treatment challenge. Follow-up studies are needed to better understand the clinical course and determine appropriate management for such patients.

\section{Disclosure}

Authors report no conflict of interest.

\section{References}

1. Alba AA, Lawton MT. Anterior cerebral artery bypass for complex aneurysms: an experience with intrcranial-intracranial reconstruction and review of bypass options. J Neurosurg 2014; 120 : 1364-1377.

2. Chang HW, Youn SW, Jung C, Kang S, Sohn CH, Kwon BJ, Han MH. Technical strategy in endovascular treatment of proximal anterior cerebral artery aneurysms. Acta Neurochir 2011; 153: 279-285.

3. Day AL, Gaposchkin CG, Yu CJ, Rivet DJ, Dacey Jr RJ. Spontaneous fusiform middle cerebral artery aneurysms: characteristics and a proposed mechanism of formation. J Neurosurg 2003; 99 : 228-240.

4. Dunn G, Gerrard J, Jho D, Ogilvy C. Surgical treatment of a large fusiform distal anterior cerebral artery aneurysm with in situ endto-side A3-A3 bypass graft and aneurysm trapping: case report and review of the literature. Neurosurgery 2011; 68: E587-591.

5. Echiverri HC, Rubino FA, Gupta, SR, Gujrati M. Fusiform aneurysms of vertebrobasilar arterial system. Stroke 1989; 20: 1741-1747.

6. Endo T, Mori K, Maeda M. Multiple arteriosclerotic fusiform aneurysms of the superficial temporal artery. Neurol Med Chir 2000; 40: 321-323.

7. George KG, Rennie A, Saxena A. Multiple cerebral aneurysms secondary to cardiac myxoma. Br J Neurosurg 2012; 26: 409-411.

8. Hori N, Takahashi N, Furuichi S, Mori K, Onizuka M, Tsutsumi K, Shibata S. Giant fusiform aneurysms in the middle cerebral artery presenting with hemorrhages of different origins. J Neurosurg 2003; 99: 391-396

9. Jing L, Liu J, Zhang Y, Paliwal N, Meng H, Wang S, Yang X. Analysis of Multiple Intracranial Aneurysms with Different Outcomes in the Same Patient After Endovascular Treatment. World Neurosurgery 2016; 91: 399-408.

10. Juvela S. Risk factors for multiple intracranial aneurysms. Stroke 2000; 31: 392-397.

11. Krings T, Mandell DM, Kiehl TR, Geibprasert S, Tymianski M, Alvarez $\mathrm{H}$, terBrugge $\mathrm{KG}$, Hans FJ. Intracranial aneurysms: from vessel wall pathology to therapeutic approach. Nat Rev Neurol 2011; 7 : 547-559.

12. Nobutaka H Nobuaki T, Shoji F, Katshuharu M, Masanari O, Keisuke T, Shobu S. Giant fusiform aneurysms in the middle cerebral artery presenting with hemorrhages of different origins. J Neurosurg 2003; 99: 391-396.

13. Park S, Yim, M, Lee C, Ealmaan Kim E, Son E. Intracranial Fusiform Aneurysms: It's Pathogenesis, Clinical Characteristics and Managements. J Korean Neurosurg 2008; 44: 116-123.

14. Wakabayashi Y, Nakano T, Isono M, Shimomura T, Hori S. Dissecting Aneurysm of the Anterior Cerebral Artery Requiring Surgical Treatment: Case Report. Neurol Med Chir 2000; 40: 624-627. 